\title{
Freeze-Etching: Freezing Velocity and Crystal Size at Different Locations in Samples
}

\author{
G. E. P. M. VAN VENROOIJ, A. M, H. J. AERTSEN AND W. M. A. HAX \\ Medical Physics Department, State University Utrech, the Netherlands \\ P. H. J. T. VFRVERGATRT AND J. J. VERHOEVEN \\ Binlogical Ultrastructure Research Unit, Sate University Ulreht, The Nelherlands \\ AND \\ H. A. VAN DER VORST \\ Computer Centre, State University Utrecht, The Netherlands
}

The structural and biochemical integrity of biological material can seriously be damaged during the freezing procedure. Previous studies have revealed that not only the mechanical disruptive power of ice crystals but also phenomena like osmotic stress, precipitation of salts, segregation of macromolecules, and small cytoplasmic organelles will inflict injury to the cell $(12-16,23)$. Only cells which are frozen very rapidly are well preserved $(2,13,14$, 16). Such quenching techniques are employed in freeze-etching. In an early freezeetch paper it was shown by Moor (16) that cells frozen at slower rates exhibited a cytoplasm with large smooth areas surrounded by course material in freeze-fractured replicas. It was suggested that the smooth areas represent fracture faces of ice crystals and that these are girdled by cytoplasmic material. A considerable deerease in the size of the ice crystals and a concomitant improvement of the viability upon thawing could be achieved by addition of cryoprotectants like glycerol or dimethyl sulfoxyde $(13,14)$ to the malerial before quenching. Good preservation of the cell's

Received April 17, 1974. ultrastructure can be obtained by the addition of cryoprotectants; by the application of ultrarapid quenching techniques (1); or by the freezing of cells under pressure (18).

From an experimental and numerical analysis of the freezing process Riehle (18) concluded that biological systems can only be vitrified when the cooling velocity is higher than $10,000^{\circ} \mathrm{C} / \mathrm{s}$. It was assumed that in the center of a specimen cooling velocity will be lowest and crystal size greatest. By applying a high pressure he could obtain "vitrification" of $5 \%$ glycerol solutions, which was concluded from freezectch replicas showing amall 50-100 $\AA$ "ice crystals." He found that the erystal size decreased approximately with the square of the cooling time.

The aim of the present report is a better understanding of the relation between crystal size, freezing velocity, and location in the specimen. Therefore, we compared numerical and experimental data on freezing velocity with crystal size measurements from freeze-etch replicas.

The theoretical analysis of the cooling process was performed for simplicity on a spherical model. Apart from a few exact 
solutions for infinite slabs all quantitative analyses of the temperature changes in systems in which phase changes occur have to be attempted either by approximation. methods or by numerical methods. $\mathrm{Nu}-$ merical procedures have been given for circular cylinders $(4,11)$ and spheres $(9,10,20)$, however, in these cases special fixed boundary or initial conditions are required. We used the method of finite differences. The advantages of this method are that arbitrary choices of boundary and initial conditions are allowed and that by simple change of a few equations solutions for infinite slabs and infinite circular cylinders can also be calculated.

\section{MATERIALS AND METHODS}

\section{Temperature Measurements}

Experimental results were obtained using a thin-walled $(100 \mu \mathrm{m})$ silver cylinder with a diameter of $2 \mathrm{~mm}$ and a height of $2 \mathrm{~mm}$, constructed by carefully piercing a little silver rod. This cylinder was filled with a $5 \%$ glycerol solution. The change in temperature due to immersion in Freon 22 $\left(-145^{\circ} \mathrm{C}\right)$ was measured using a thermocouple with a diameter of $100 \mu \mathrm{m}$, whereas the leads have diameters of $50 \mu \mathrm{m}$. The thcrmocouple was positioned under microscopic control in the cylinder by putting the leads through opposite holes $(100 \mu \mathrm{m})$ in the wall. By bending the leads outside the cylinder wall the thermocouple was more or less fixed. After the measurement the cylinder content was thawed in order to check that the positioning of the couple was not disturbed during quenching in the freon. In this way the change in temperature was measured at different locations in the plane in the middle of the cylinder perpendicular to the axis: in the center, near the border and halfway between the center and the border.

\section{Electron Microscopy Freeze-Fracturing}

A conventional cylinder of $1-\mathrm{mm}$ diameter was filled with a $20 \%$ glycerol solution and a small hollow, open cylinder was placed on top of it; the complete speeimen holder was quenched in Freon 22, and then transferred to a Denton freeze-etch machine. The specimen was frictured at $-100^{\circ} \mathrm{C}$; etched for $2 \mathrm{~min}$, and subsequently $P t / C$ shadowed. Only those replicas displaying the complete fracture area of the cylinder were used.

Other electronmicrographs were also made from preparations spray-frozen according to the Bachmann-method (1).

Electronmicrographs were made on a Siemens Elmiskop IA.

\section{Crystal Dimensions}

Crystal dimensions were measured by means of simple stereological procedures (21). In the area in which the dimensions of the crystals were to be measured two test lines were drawn with known length $\lambda$ : one in the radial direction and one in the direetion perpendicular to the radial direction (this line has the form of the arc of a circle). The number of intersections $P$ were counted. So the average lengths of the crystals as they display in that area are given by $\lambda / P$. Because we use two test lines crystal measures are known then in two perpendicular directions. The length of the radial test line was chosen to be short compared to the radius of the sample.

\section{THEORY}

\section{Model}

The samples used for freeze-etching have the form of a circular cylinder with a diameter which is almost equal to the height. For reasons of simplifications in the theoretical and numerical analysis a sphere will be considered (see discussion). It will be assumed that the sphere has radius $R_{1}$ and consists of a liquid. At time $t=0$ the temperature of the sphere is $T_{1}\left(T_{1}>T_{f}\right.$ with $T$, as the freezing point). The sphere is surrounded by a boundary layer with thickness $R_{2}-R_{1}$, which has an initial temperature of $T_{2}\left(T_{2}<T_{f}\right)$. The layer has 




FIG, 1. Freezing of the sphere. $x(t)$ denotes the location of the boundary between the liquid and the solid phase at time $t$. Outside $R_{2}$ : cooling liquid which remains at temperature $T_{2}$. The shell between $R_{1}$ and $R_{2}$ : cooling liquid with initial temperature $T_{2}$. The shell between $x(t)$ and $R_{1}$ : solid phase. The sphere within $x(t)$ : liquid phase.

heat transfer properties of the cooling liquid. The outside of the boundary layer is assumed to stay at temperature $T_{2}$ during the cooling process. During the phase transition heat of crystalization is liberated at the boundary between the liquid and solid phase (see Fig. 1). This boundary will move from the surface of the sample to its center.

\section{Symbols and definitions}

$t=$ time.

$r \quad=$ radial distance measured from the center of the sphere.

$T(r, t)=$ temperature at time $t$ and position $r$.

$T_{f} \quad=$ freezing point.

$x(t) \quad=$ location of the boundary between liquid and solid phase at time $t$, measured from the center of the sphere.

$\rho \quad=$ density.

c. = specific heat.

$K$ = heat conductivity $\tilde{\overline{\bar{D}}}$ the quantity of heat which is transmitted as a result of unit temperature difference per unit time through a plate of unit thickness across an area of unit surface.

$k=$ diffusivity of heat $\overline{\bar{D}}(K / \rho c)$.

$L \quad=$ crystallization heat. $l, s, c=$ indices, referring to liquid, solid phase, and cooling liquid, respectively.

$V_{f}=$ the freezing velocity of a volume element $d V$ defined as the rate of change of temperature $T$ with time $t$ immediately after solidification.

\section{Heat Conduction Equations}

Cooling by means of diffusion is described for spherical symmetry by the equation:

$$
\begin{aligned}
& \left(\partial^{2} T / \partial r^{2}\right)+(2 / r)(\partial T / \partial r) \\
& =(1 / k)(\partial T / \partial t) .
\end{aligned}
$$

During the freezing process a transition boundary separating the liquid and solid phase moves inwards through the sphere. The rate of change of unsolidified volume depends on the heat balance at the boundary according to

$$
\begin{array}{r}
L_{\rho} \frac{d\left(\frac{4}{3} \pi x^{3}\right)}{d t}=-4 \pi x^{2} K_{1} \frac{\partial T_{1}}{\partial r} \int_{r=x} \\
+4 \pi x^{2} K_{\varepsilon} \frac{\partial T_{s}}{\partial r} / .
\end{array}
$$

Furthermore, it holds that

$$
T_{*}(x)=T_{1}(x)=T_{f}
$$

\section{Boundary Conditions}

The temperature at the center of the sphere $(r=0)$ satisfies the condition:

$$
(\partial T / \partial r)=0 \text {. }
$$

At the surface of the sphere $\left(r=R_{1}\right)$ there is no heat source or sink, so,

$$
K_{\mathrm{a}} \frac{\partial T_{c}}{\partial r} /_{r=R_{1}}=K_{q} \frac{\partial T_{q}}{\partial r} \overbrace{r=R_{1}}
$$

in which the index $q$ refers to the liquid $\left[T\left(R_{1}\right)>T_{j}\right]$ or the solid phase $\left[T\left(R_{1}\right)\right.$ $\left.<T_{f}\right]$. 
TABLE 1

Values for the Used Material Constants

\begin{tabular}{lrccc}
\hline Quantity & $\begin{array}{c}\rho \\
\left(\mathrm{kg} \mathrm{m}^{-1}\right)\end{array}$ & $\left(\mathrm{J} \mathrm{kg}^{c}{ }^{\circ} \mathrm{C}^{-1}\right)$ & $\left(\mathrm{J}^{-1} \mathrm{~m}^{-1}{ }^{\circ} \mathrm{C}^{-1}\right)$ & $\begin{array}{c}k \\
\left(\mathrm{~m}^{2} \mathrm{~s}^{-1}\right)\end{array}$ \\
\cline { 1 - 2 } Water & 1000 & 4200 & 0.588 & $14 \times 10^{-8}$ \\
Ice & 900 & 2100 & 2.52 & $13 \times 10^{-7}$ \\
Freon 22 & 1500 & 1260 & 0.126 & $\mathbf{6} \times 10^{-8}$ \\
\hline
\end{tabular}

Assuming intimate contact at the surface of the sphere we have in addition to Eq. (5),

$$
T_{s}\left(R_{1}\right)=T_{q}\left(R_{1}\right) .
$$

From the model assumptions it follows that the temperature at $r=R_{2}$ is given by:

$$
T\left(R_{2}, t\right)=T_{2}
$$

\section{Initial Conditions}

At the time that cooling starts $(t=0)$ the temperatures are supposed to be given by:

$$
\left.\begin{array}{l}
T=T_{1}>T_{f} \text { for all values of } r<R_{1} \\
T=T_{2}<T_{f} \text { for all values of } r>R_{1} \\
T=\frac{K_{q} T_{1}+K_{t} T_{2}}{K_{q}+K_{0}} \text { for } r=R_{1}
\end{array}\right\}
$$

The numerical treatment of the cooling process is evaluated in Appendix 1. The accuracy of the obtained solution appears to be about $5 \%$, which will be illustrated in Appendix 2.

\section{RESUITS}

\section{Material Constants}

The calculations have been carried out for a system consisting of water (cq. ice) and Freon 22, as these correspond to the experimental conditions. The values for the material constants are listed in Table 1.

Other values used in the calculations are listed in Table 2.

\section{Temperature Measurements}

The temperatures in a cylinder (diameter $2 \mathrm{nmm}$ ) were measured using a thermocouple. This was done at three different locations: in the center $(r=0 \mathrm{~mm})$, half-way between the center and the outersurface $(r=0.5 \mathrm{~mm})$, and near the border $(r \lesssim 1.0 \mathrm{~mm})$. The results of these measurements are shown in Fig. 2a.

\section{Theoretical Results}

Different definitions for freezing velocity eq. cooling rate appear in the literature. In this report we define freezing velocity as the decrease in temperature per unit time immediately after the moment that freezing has started. At this temperature the growth rate of crystals will in general be the fastest (18).

Measurements during which only the thermocouple was immersed in Freon 22 show that the cooling of the thermocouple from 20 to $-145^{\circ} \mathrm{C}$ takes place within $0.01 \mathrm{~s}$. Because this cooling velocity is very high compared to the measured cooling velocities, it is not likely that the conduction of heat by the leads of the thermocouple will disturb the measurements. However, the thermocouple has a diameter of $100 \mu \mathrm{m}$, so the temperature indicated by the thermocouple belongs to an area of as least $100 \mu \mathrm{m}$. We have tried to simulate thit during the numerical treatment on the cooling process of the sphere by averaging the temperatures within an area of about $100 \mu \mathrm{m}$. The value of $R_{1}$ was taken equal to $1.145 \mathrm{~mm}$ (see discussion) and $R_{2} / R_{1}$ was taken equal to $62 / 60$. The results are given in the Figs. $2 \mathrm{~b}$ and $2 \mathrm{c}$. In Fig. $2 \mathrm{~b}$ the freezing point was chosen $-15^{\circ} \mathrm{C}$ and in Fig. $2 \mathrm{c}$ equal to $-1^{\circ} \mathrm{C}$. (The freezing point of a $5 \%$ glycerol solution is about $-1^{\circ} \mathrm{C}$ ).

TABLE 2

\begin{tabular}{cc} 
Values Usho for Several Used Constants \\
\hline Quantity & \multicolumn{1}{c}{ Value } \\
\hline$T_{1}$ & $+15^{\circ} \mathrm{C}$ \\
$T_{2}$ & $-145^{\circ} \mathrm{C}$ \\
$l$ & $4200 \mathrm{~J} \mathrm{~kg}^{-1}$ \\
$\alpha$ & 0.3 \\
\hline
\end{tabular}




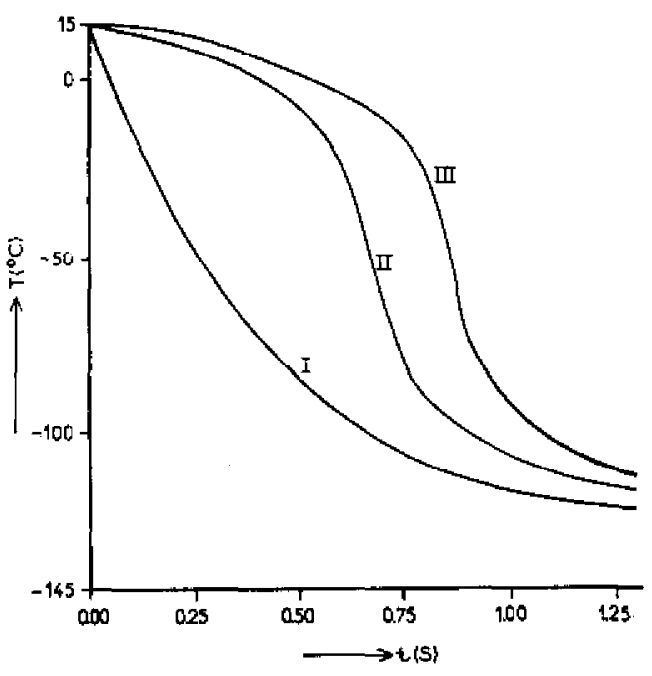

FIg. 2a. Fxperimental results of temperature measurements in a cylinder with a diameter of $2 \mathrm{~mm}$ and a height of $2 \mathrm{~mm}$. The three curves correspond with three different locations of the thermocouple: (I) near the border $(r=1.0 \mathrm{~mm}$ ), (II) half-way between the center and the border $(r=0.5 \mathrm{~mm})$; (III) in the center $(r=0.0 \mathrm{~mm})$.

Figures $2 \mathrm{a}, 2 \mathrm{~b}$, and $2 \mathrm{c}$ show that there exists a fair agreement between the model calculations and the experimental results.



Fig. 2b. Theoreticel result for the cooling process of a sphere with radius $R_{1}=1.145 \mathrm{~mm}$ filled with water immersed in Freon 22. The curves correspond with three different locations: (I) near the border ( $r=R_{1}$ ); (II) halfway between the center and the border. $\left(r=0.5 R_{1}\right)$; (III) in the center $\left(r=0.0 R_{1}\right)$. $T_{r}$ is chosen equal to $-15^{\circ} \mathrm{C}$.
Note that the freezing velocity is clearly higher in the center than halfway between the outer surface and the center.

\section{Crystal Sizes}

By using the complete fracture area of a cylinder with a diameter of $1 \mathrm{~mm}$ filled with a $20 \%$ glycerol solution we were able to compare the crystal sizes at different locations. Figures $3 \mathrm{a}, 3 \mathrm{~b}$, and $3 \mathrm{c}$ obviously show that the erystals in the center and near the border are smaller than the crysstals in the other fracture domain. Staehelin and Bertaud (19) also mention an increasing crystal size when measuring from surface to interior of a frozen fractured glycerol water droplet. However, their findings only partly agree with ours, as they don't mention the decreasing crystal size in a particular area around the center.

Besides, from $\mathrm{Eq}$. (1) it follows that reduction of the dimensions of the object with a factor $\gamma$ will result in an increase of the cooling rate by a factor $\gamma^{2}$ on corresponding sites. A simple dimensional analysis suggests that the thickness of the boundary layer will also be reduced by $\gamma$. Figure 3d shows the small crystal sizes ob-

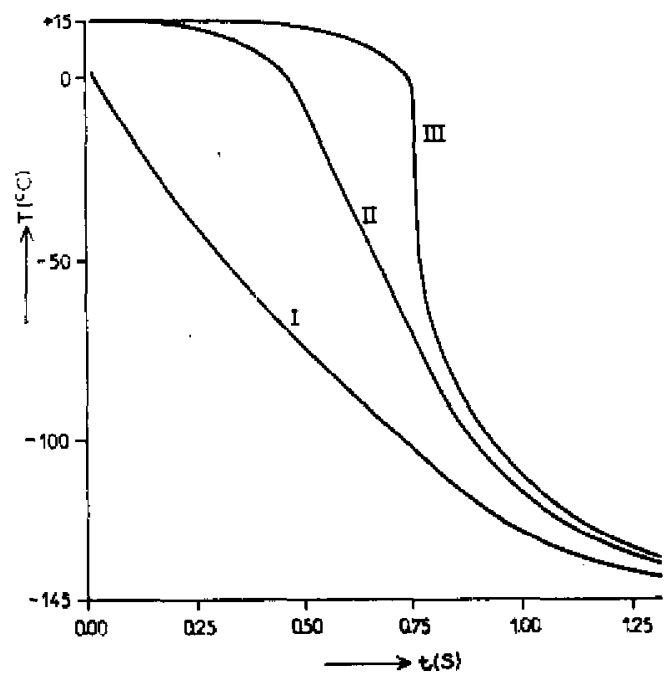

Fig. 2c. Idem as in Fig. 2b, only $T_{f}$ is chosen equal to $-1^{\circ} \mathrm{C}$ in this case. 
tained in a spray-frozen preparation according to the Bachmann-method. In this case the object size is about $10-20 \mu \mathrm{m}$.

Within the replica (diameter $1 \mathrm{~mm}$ ) we also measured the dimension $\phi$ of the erystats at different locations. Using the numerical scheme the freezing velocities at different locations within a sphere with $R_{1}=0.5725 \mathrm{~mm}$ (see discussion) and $R_{2} / R_{1}=62 / 60$ were calculated on corresponding sites. The results of these measurements and calculations are shown in Fig. 4. (now the temperatures are not averaged over an area of $100 \mu \mathrm{m}$ ).

The ratio $\beta=R_{2} / R_{\mathrm{I}}$ determines the width of the boundary layer of cooling liquid surrounding the sphere in which the temperature does not remain at its initial temperature. The parameter $\beta$ may be considered as a measure for convection in the cooling liquid. $\beta=\infty$ suggests no convection, whereas $\beta=1$ indicates that all heat is removed by convection.

To illustrate that the width of the boundary layer plays an important role, the calculations on the sphere $\left(R_{1}=1.145 \mathrm{~mm}\right)$ were repeated for a slightily different value of



FIG. 3. a: Crystals from the center. Bar $=1 \mu \mathrm{m}$. $\mathrm{b}$ : Crystals from an area halfway between the center and the border. Bar $=1 \mu \mathrm{m}$. $\mathrm{c}$ : Crystals near the border. Bar $=1 \mu \mathrm{m}, \mathrm{d}$ : Crystals in a spray-frozen preparation. $\mathrm{B} a \mathrm{r}=500 \AA$.

$R_{2} / R_{1}$, namely $63 / 60$. Figure 5 shows the temperatures within the sphere at the same locations as in Figs. $2 \mathrm{~b}$ and $2 \mathrm{c}$. In this case $T_{f}=-15^{\circ} \mathrm{C}$. The fact that in the rase of Freon 22 the ratio appears to be $62 / 60$

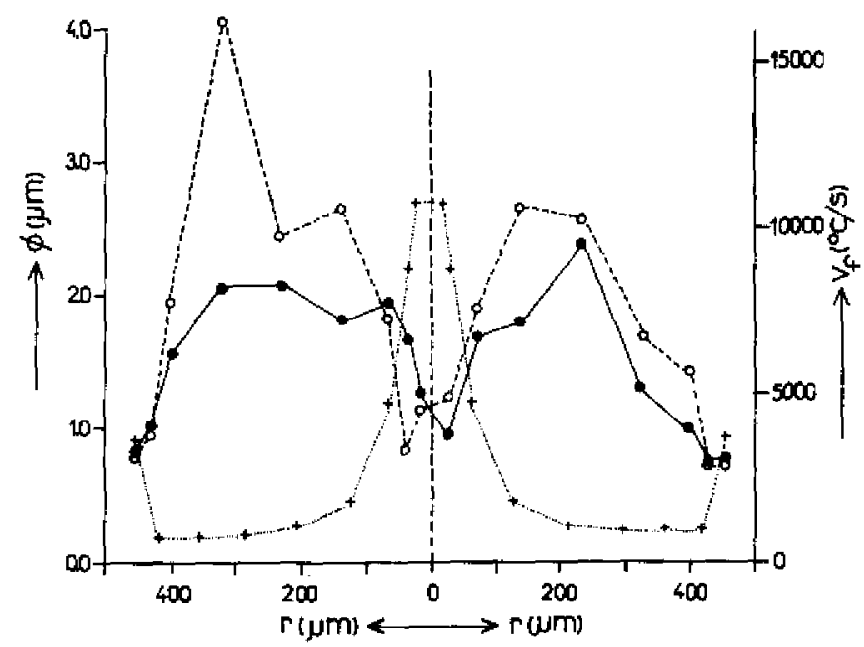

FIG. 4. Crystal sizes and freezing velocities as a function of the location within the replica with a diameter of $1 \mathrm{~mm}$. The open dots represent the length of the crystals in the radial direction, whereas the blark dots represent the length in the direction perpendicular to the radial direction. The left vertical axis indicates the value of these dimensions. The erosslets represent the freezing velocities calculated from the results of the numerical scheme applied to the freezing sphere (diameter: $1.145 \mathrm{~mm}$ ) on corresponding sites. The right vertical axis indicates the velocities. 


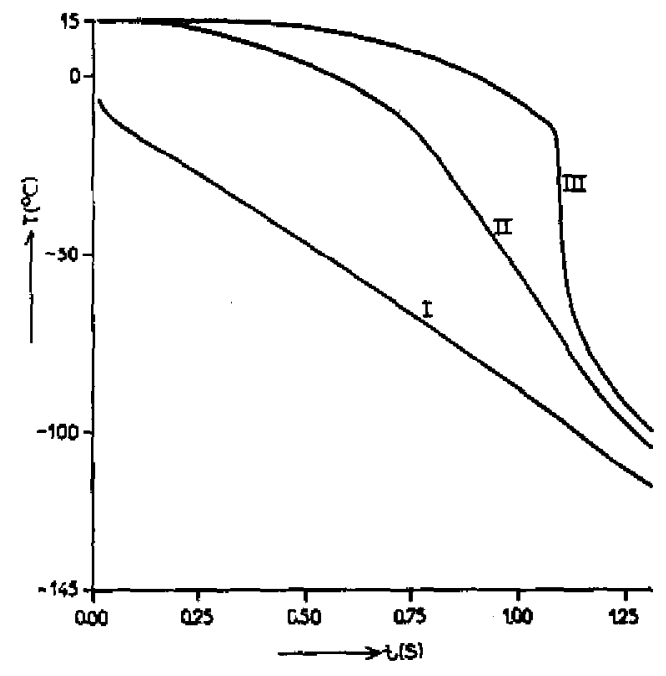

FIG. 5. Idem as in Fig. 2b only $N_{2}=63$ and $N_{1}=60$.

implies a reasonable convection within the cooling liquid.

\section{Influence of Freezing Point upon Freezing Velocity}

Caleulating freezing velocities near the border and in the center of the sphere (now without averaging over an area of $100 \mu \mathrm{m}$ ) gives an impression of the influence of $T_{f}$ upon the freezing velocity $V_{f}$ within the sample (see Table 3 ).

\section{DISCUSSION}

\section{Difference in Geometry}

Although in the actual experiments the sample is a finite eylinder rather than a sphere, the sphere has been taken as a model because of the difficulties associated with the mathematical treatment of heat

TABLE 3

Fremzing Veiocities Near the Surface and IN ThF Center of the SPHere for Two Values of $T_{t}$. Note: Drameter Sample $=2 \mathrm{~mm}$ )

\begin{tabular}{lrl}
\hline & $T_{f}=-15^{\circ} \mathrm{C}$ & $T_{f}=-1^{\circ} \mathrm{C}$ \\
\hline$V_{f}$ in center & $2000^{\circ} \mathrm{C} \mathrm{s}^{-1}$ & $2200^{\circ} \mathrm{C} \mathrm{s}^{-1}$ \\
$V_{f}$ near surface & $200^{\circ} \mathrm{C} \mathrm{s}^{-1}$ & $1000^{\circ} \mathrm{C} \mathrm{s}^{-1}$ \\
\hline
\end{tabular}

diffusion in a finite cylinder. The dimensions of the cylinder (height $=$ diameter) more or less justify this approximation.

In order to obtain an optimal similarity the radius of the sphere was made equal to 1.145 times the radius of the cylinder. In that case the volume of the sphere is equal to the volume of the cylinder. Williamson and Adams (22) calculated the time dependence of the central temperature during cooling of a sphere and of a cylinder of length equal to diameter from $T_{\mathrm{v}}$ to $0^{\circ} \mathrm{C}$ (no freezing occurs and the border stays at $0^{\circ} \mathrm{C}$ ). Recalculation of these temperatures for a sphere and cylinder with equal volumes shows that the central temperature of the sphere and the cylinder are much the same (see Fig. 6).

\section{The Density}

In the model calculations the volume change upon solidification is neglected. Such changes cause an increase in pressure within the sample, which in turn leads to a lowering of the freezing point. Experiments suggest that the pressure is allowed to escape through cracks in the ice crust, possibly giving rise to long ice crystals in the radial direction like shown in Fig. 7 .

Experiments by Riehle (18) suggest that the pressure necessary to show an appreciable effect on the crystal sizes is very high $(100-1000 \mathrm{~atm})$. It is hardly conceivable that pressures of this magnitude are established inside the sample. Furthermore,

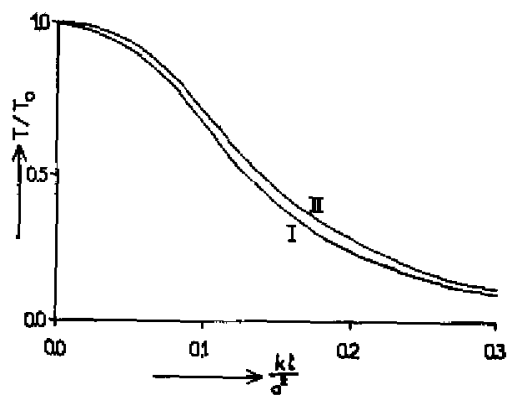

FIg. 6. Central temperature in (I) cylinder with diameter equal to height and (II) sphere. The radius of the cylinder is $a$ and that of the sphere $1.145 a$. 


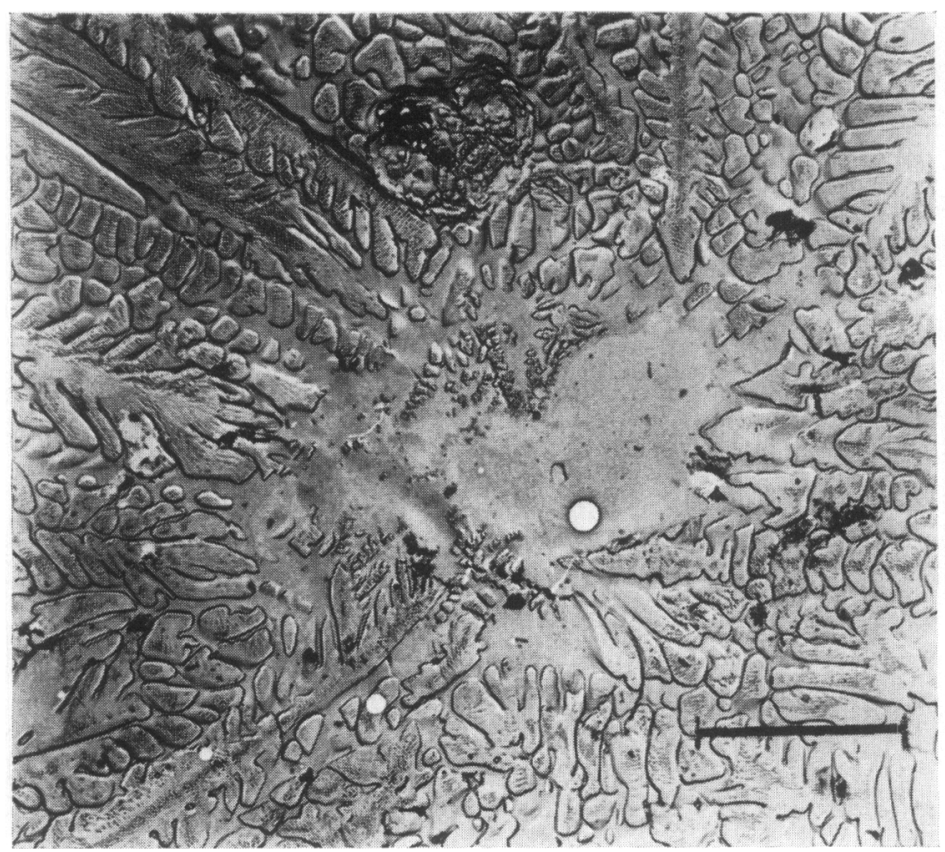

Fig. 7. Electrunmicrograph from a freeze-etch replica showing the center area of a frozen sample (diameter cylinder $1 \mathrm{~mm}$ ). Note the radial pressure escape routes. Bar $=5 \mu \mathrm{m}$.

in studies concerning heat transfer in one dimension Heitz and Westwater (8) show that the effect of unequal phase densities upon the interfacial velocity is small (in the order of $5-10 \%$ ).

\section{Freezing Temperature $\mathrm{T}_{f}$}

Due to the lowering of the freezing point and segregation phenomena in the freezing region no exact value for the freezing point is known. From the theoretical analysis, however, we know that there is a sharp transition in the time-dependent course of the temperature at the freezing point due to the changes in heat transfer properties at, that point (Fig. 2b). This change favors the diffusion of heat from the boundary between solid and liquid phase to the cooling liquid, and thus the cooling rate inereases at that point. In the experimental curve for the central temperature (Fig. 2a) this occurs at about $-15^{\circ} \mathrm{C}$. On the other hand the freezing point of a $5 \%$ glyeerol solution is about $-1^{\circ} \mathrm{C}$. The fact that in Fig. 2a the central freezing point is $-15^{\circ} \mathrm{C}$ may be due to supercooling or to an accumulation of glycerol in front of the moving phase transition. From Table 3 it follows that in our case using the initial conditions mentioned the value of $T_{f}$ strongly influences the freezing velocities near the border of the sample and only slightly in the center (this effect is obscured in the Figs. $2 \mathrm{~b}$ and $2 \mathrm{c}$ by averaging the temperatures over an area of about 100 $\mu \mathrm{m})$. Because of the fact that near the surface the freezing point will indeed be $-1{ }^{\circ} \mathrm{C}$ the freezing velocities calculated using $T_{f}=-1^{\circ} \mathrm{C}$ will be more realistic than those calculated under the assumption that $T_{f}=-15^{\circ} \mathrm{C}$. For this reason the freezing velocities in Fig. 4 are calculated under the condition $T_{f}=-5^{\circ} \mathrm{C}$, corresponding to the freezing point of a $20 \%$ glycerol solution.

\section{Differences in Dimensions and Solutions}

Since the temperature measurements were carried out using a thermocouple with a diameter of $100 \mu \mathrm{m}$ the diameter of 
the cylinder could not be taken too smalk in order to measure local temperatures. A diameter of $2 \mathrm{~mm}$ seemed a reasonable value. The cylinder was filled with a $5 \%$ glycerol solution. Thus, it would be logical to use for freeze-etehing a cylinder with a diameter of $2 \mathrm{~mm}$ with a $5 \%$ glycerol solution to get a replica displaying the complete fracture area. However, such a replica could not be kept undamaged during the cleaning procedure. It was necessary to leduce the diameter to $1 \mathrm{~mm}$ and to increase the glycerol concentration to $20 \%$ in order to obtain a stable replica.

\section{Wall Thickness}

The wall thickness of the silver cylinders used is about $100 \mu \mathrm{m}$, but silver has a thermal conductivity which exceeds those of water and Freon 22 by about a factor 1000. This means that the effective wall thickness is negligible compared to the sample diameter.

\section{Freezing Velocity and Crystal Size}

From Fig. 4 a difference can be observed between the relation of freezing velocity and crystal size near the border as compared to that at the center. This may be due to convection phenomena. At the start of the cooling process the sample will move with a high speed through the cooling liquid. Also in this part of the cooling process, the temperature differences are largest. At this time the boundary layer around the sample will be very thin, resulting in high cooling rates near the border. In the theoretical analysis a boundary layer with constant thickness is considered.

From Fig. 4 it became evident that the dimensions of the crystals in the radial direction are larger than those in the direction perpendicular to this direction. This may be due to the direction along which the pressure is released (see also Fig. 7).

We want to emphasize that the measured dimensions in Fig. 4 are the dimensions of the crystals as they appear in the replica.
The average crystal size in the sample will be higher (6).

\section{Inplications for Freezing Specinens}

According to Moor (17) specimens can only be solidified in the native state without changes in structure or chemical compositions if the temperature interval from 0 to $-100^{\circ} \mathrm{C}$ is passed in less than $0.01 \mathrm{~s}$. This is equivalent to a freezing velocity of about $10,000^{\circ} \mathrm{C} / \mathrm{s}$.

From the results in Fig. 4 it is found that the lowest freezing velocity in a sample with a diameter of $1 \mathrm{~mm}$ is about $800^{\circ} \mathrm{C} / \mathrm{s}$. Since a reduction of the object dimensions by a factor $\gamma$ will roughly result in an increase of the freezing velocities by a factor $\gamma^{2}$, it is to be expected that samples with diameters less than $0.3 \mathrm{~mm}$ can be frozen in such a manner that the freezing velocity everywhere in the sample is higher than $10,000^{\circ} \mathrm{C} / \mathrm{s}$. In samples with diameters between 0.3 and $1.0 \mathrm{~mm}$ there will be an area around the center in which the freezing velocities are higher than $10,000^{\circ} \mathrm{C} / \mathrm{s}$. These findings agree roughly with those of Riehle (18) who found that specimen with dimensions less than $100 \mu \mathrm{m}$ can be solidified in the native state.

\section{CONCLUSIONS}

There exists a fair agreement between the temperature course in a cylinder measured with a thermocouple and the temperature course as calculated on corresponding sites in a sphere.

The freezing velocity depends on the location in the sample. From experimental and theoretical results it follows that the freezing velocity is higher at the border and in the center of the sample than half way between the center and the outer surface of the sample.

Flectronmicrographs show that the erystals in the center and near the border are smaller than halfway between the center and the outer surface of the replica. 
The reduction in the dimensions of the sample will increase the freezing velocities at corresponding sites proportional to the square of the reduction. From this and from theoretical results it is to be expected that samples with a diameter less than $0.3 \mathrm{~mm}$ can be frozen without changes in structure and chemical properties. In samples with diameters between 0.3 and $1.0 \mathrm{~mm}$, there will be an area around the center in which the substance is frozen without these changes.

\section{SUMMARY}

Freezing velocity was studied experimentally as well as theoretically in relation to sample size and location within the sample. Crystial sizes in a freeze-etch replica displaying the complete fracture area of a sample were compared to the experimental and theoretical results concerning freezing velocities at different locations in such a sample. The freezing velocity was found to be higher in the center and near the surface than halfway between the center and the outer surface of the sample. Also, the crystals in the center and near the border appear to be small as compared to the crystals in the other fracture domain.

\section{APPENDIX 1}

\section{Numerical Methods}

\section{Symbols and Definitions}

$N_{2}=$ number of shells in the sphere together with the boundary layer.

$N_{1} \quad=$ number of shells in the sphere.

$\Delta r \quad=$ thickness of one shell.

$n \quad=$ shell number

$T(n)=$ temperature at time $t$ at location $r=n \Delta r$.

$T^{+}(n)=$ temperature at time $t+\Delta t$ at location $r=n \Delta r$.

$x(t)=$ position of the boundary between liquid and solid phase at time $t$.

$x^{+}(t)=$ position of the boundary at time $t+\Delta t$.

$y=$ shell number in which the boundary is moving $\left(y=1,2, \cdots N_{1}\right)$.

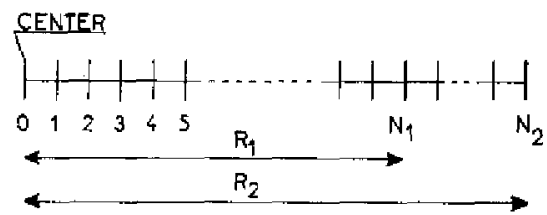

FIG. 8. Division of the sphere together with the boundary layer into shells of equal width. The shells are numbered as indicated. The sphere consists of $N_{1}$ shells and the boundary layer of $N_{2}-N_{1}$ shells.

$p \quad=$ position of the boundary with respect to $y$ at time $t$.

$p^{+} \quad=$ position of the boundary with respect to $y$ at time $t+\Delta t$.

$\alpha \quad=\left[k t /(\Delta r)^{2}\right]$.

\section{Numerical Scheme}

The sphere together with the boundary layer are divided into $N_{2}$ shells of equal thickness $\Delta r$. The shells are numbered as indicated in Fig. 8.

Using the tmethods of finite differences the derivatives of the temperature $T$ at position $n(r=n \Delta r)$ are given by

$$
\begin{aligned}
& \frac{\partial T}{\partial r}{ }_{n}=\frac{T(n+1)-T(n-1)}{2 \Delta r} \\
& +O\left[(\Delta r)^{2}\right], \\
& \frac{\partial^{2} T}{\partial r^{2}} /=\frac{T(n+1)-2 T(n)+T(n-1)}{(\Delta r)^{2}} \\
& +O\left[(\Delta r)^{2}\right],
\end{aligned}
$$

$\frac{\partial T}{\partial t} /=\frac{T+(n)-T(n)}{\Delta t}+O\left[(\Delta t)^{2}\right]$.

Using these expressions and neglecting second and higher differences, the heat equation Fq. (1) takes the form:

$$
\begin{aligned}
& T^{+}(n)=T(n)+\left[k \Delta t /(\Delta r)^{2}\right] \\
& \quad \times\{[(n+1) T(n+1)-2 n T(n) \\
& \quad+(n-1) T(n-1)] / n\}(n \neq 0) .
\end{aligned}
$$

For $n=0$ it holds that (3)

$$
\begin{aligned}
T^{+}(0)=T(0)+ & {\left[6 k \Delta t /(\Delta r)^{2}\right] } \\
& \times\{T(1)-T(0)\} .
\end{aligned}
$$



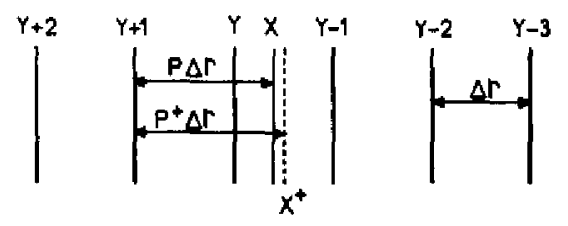

SOLID

LIOUID

FIG. 9. Position of the moving boundary in shell number $y$.

Now if the method has to be useful, errors must not grow. The required restriction on $\Delta t$ (the stability condition) is given by $(3)$ :

$$
\alpha=\left[k \Delta t /(\Delta r)^{2}\right] \leqslant \frac{1}{2}
$$

To obtain a time step admissable in all regions the maximum value for $k$ of the three values $k_{s}, k_{r}$ and $k_{l}$ must be taken.

Note. 1. Equations (9) and (10) are only valid if the same value for $k$ holds for the position $n+1, n$ and $n-1$.

To calculate the position $x(t)$ of the moving boundary a similar approach as given by Crank (1957) for calculations on plane systems is followed. Let the boundary at time $t$ be somewhere in the layer between the positions $y$ and $y-1$, such that its position $x$ is given by $x=(y+1-p) \Delta r$, where $p$ is fractional and $1<p<2$. At time $t+\Delta t$ the position is given by: $x^{+}=\left(y+1-p^{+}\right) \Delta r$. See also Fig. 9 .

Taking into account the spherical structure of the model, Eq. (2) can be approximated by:

$$
\begin{gathered}
L_{\rho} \frac{4 \pi}{3}(\Delta r)^{3} \cdot\left\{\frac{(y+1-p)^{3}-\left(y+1-p^{+}\right)^{8}}{\Delta t}\right\} \\
=K_{1}(y+1-p)^{2} 4 \pi(\Delta r)^{2} \frac{\partial T_{1}}{\partial r}{ }_{r=x} \\
-K_{d}(y+1-p)^{2} 4 \pi(\Delta r)^{2} \frac{\partial T_{s}}{\partial r} / .
\end{gathered}
$$

Or, after rearranging the terms:

$$
\begin{aligned}
p^{+}= & y+1-(y+1-p)\left\{1-\frac{3 \Delta t}{L_{\rho}(y+1-p) \Delta r}\right. \\
& \left.\times\left(K_{1} \frac{\partial T_{1}}{\partial r}{ }_{r=x}-K_{a} \frac{\partial T_{\mathrm{\varepsilon}}}{\partial r} /\right)\right\}_{r=x}^{\frac{1}{3}} \cdot(12)
\end{aligned}
$$

The temperatures $T^{+}$at the positions $y+1$ and $y-2$ can be computed using Eq. (9). For the positions $y$ and $y-1$ we use 3-points Lagrange interpolation on the temperatures $T^{+}$at the positions $x^{+}, y+1$, $y+2$, and $x^{+}, y-2, y-3$, respectively.

The temperature gradients are computed too by means of 3-points Lagrange interpolation : for $\left(\partial T_{s} / \partial r\right) / r=x$ these points are $y+2, y+1$, and $x^{+}$, and for $\left(\partial T_{1} / \partial r\right) / r=x$ they are $x^{+}, y-2$, and $y-3$.

If $p$ crosses the value 2 , which means that the boundary between liquid and solid phase moves from one shell into another, then both $y$ and $p$ are reduced by 1 and the calculations are continued with the same equations.

At the boundary between the sphere and the cooling liquid a linear approximation for the gradients $(\partial T / \partial r)$ is used in $\mathrm{Eq}$. (5) resulting in :

$$
\begin{aligned}
& T^{+}\left(N_{1}\right)=\left\{\left[K_{q} T^{+}\left(N_{1}-1\right)\right.\right. \\
& \left.\left.\quad+K_{c} T^{+}\left(N_{1}+1\right)\right] /\left(K_{q}+K_{c}\right)\right\}
\end{aligned}
$$

From $\mathrm{Eq}$. (6) it follows

$$
T^{+}\left(N_{2}\right)=T\left(N_{2}\right) \text {. }
$$

Note 2. The methods for calculating the position $p^{+}$can only be used for values $2 \leqslant y \leqslant N_{1}-2$. In the outer and inner shells of the sphere other approximations must be made.

The exceptions to the above described scheme (Notes 1 and 2) are now treated separately:

$y=N_{1}$

Freezing starts as soon as $T\left(N_{1}\right)<T_{f}$. The first value of $p$ is computed applying the nonequidistant analogon of Eq. (13) to 
the known temperatures $T\left(N_{1}+1\right), T\left(N_{1}\right)$ and $T_{j}$. Hence,

$p_{\text {start }}=1+\frac{K_{s}\left[T_{f}-T\left(N_{1}\right)\right]}{K_{e}\left[T\left(N_{1}\right)-T\left(N_{1}+1\right)\right]}$.

This results in a first position of the moving boundary:

$$
x_{\text {start }}=N_{1}+1-p_{\text {sturt }} .
$$

This means that the latent heat, liberated by freezing of the thin shell between outer surface of the sphere and position $x_{\text {start }}$ is neglected.

Now, the temperature $T^{+}\left(N_{1}\right)$ is computed by applying the nonequidistant analogon of Eq. (13) to the known temperatures $T^{+}\left(N_{1}+1\right)$ and $T_{1}$ using the known value of $p$.

The value of $p^{+}$is calculated by means of Eq. (12) in which $\left(\partial T_{s} / \partial r\right) /_{r=x}$ is approximated by

$$
\frac{\partial T_{s}}{\partial r} \underset{r=x}{/}=K_{s} \frac{T_{r}-T^{+}\left(N_{1}\right)}{(p-1) \Delta r},
$$

i.e., linear approximation.

$y=N_{1}-1$

In this case, the temperatures $T^{+}\left(N_{1}\right)$ and $T^{+}\left(N_{1}-1\right)$ are both calculated by linear interpolation on the known temperatures $T^{+}\left(N_{1}+1\right)$ and $T_{5}$.

In order to calculate $p^{+}$the value of $\left(\partial T_{1} / \partial r\right) / r=z$ in Eq. (12) is approximated by linear interpolation on the values $T^{+}\left(N_{1}+1\right)$ and $T_{r}$.

$y=2$

Because of the boundary condition $(\partial T / \partial r) / r=0=0$ a temperature $T(-1)$ $=T(1)$ can be defined, $T^{+}(1)$ can be computed then by using 3-points interpolation on the temperatures $T_{f}, T^{++}(0)$ and $T^{+}(-1)$ $=T^{+}(1)$. In this way it is found that

$$
\begin{aligned}
& T^{+}(1)=\left\{\left[T_{f}+(p-2)(p-4) T^{+}(0)\right] /\right. \\
& y=1
\end{aligned}
$$

For the computation of $T+(0)$ the expression $T(1)-T(0)$ in Eq. (10) must be replaced by $\left[T_{f}-T(0)\right] /(2-p)^{2}$. In order to calculate $p^{+}$the valuc of $\left(\partial T_{1} / \partial r\right) / r=x$ is calculated using the expression:

$$
\frac{\partial T_{1}}{\partial r} \underset{r=. .}{/}=\frac{T_{f}-T^{+}(0)}{(2-p) \Delta r} .
$$

As soon as $p^{+}$crosses the value 2 the sphere is completely frozen. After that the cooling process of the sphere will be described again by the Eqs. (9), (10), (13), and (14).

\section{APPENDIX 2}

The Accuracy of the Numerical Solutions

\section{Stability Parameter $\alpha$}

As already noted, stability of the numerical methods requires $\alpha \leq 0.5$ (Eq. 11). During the calculations $\alpha$ has been taken equal to 0.3 . In order to check that this choice is correct, some analyses were done for both $\alpha=0.3$ and $\alpha=0.1$. The results show a complete similarity.

\section{Convergence of the Numerical Solutions}

$A$ correct numerical approach will produce a solution that converges to the exact

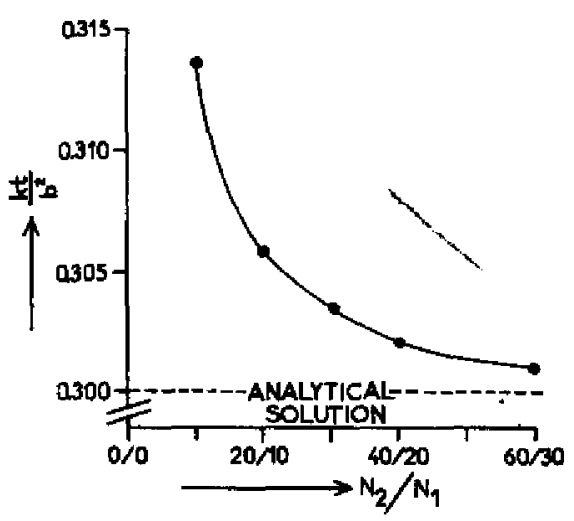

Fig. 10a. The time needed to reach the temperature $0.35 T_{0}$ at the location $r=0.2 b$ as a function of the number of shells. The dotted line indicates this time calculated using the analytical solution. 


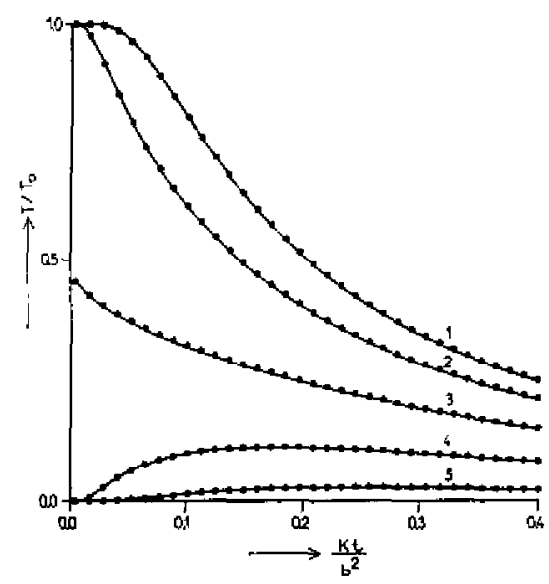

Fia. 10b. The analytical solution (line) together with the numerical solution (dots) with parameters $N_{2}=60$ and $N_{1}=30$ on different locations: (1) $r=0.2 b$; (2) $r=0.6 b$; (3) $r=1.0 b$; (4) $r=1.4 b$; (5) $r=\mathbf{1} .8 b$.

solution of the corresponding differential equation when the intervals $\Delta r$ (and thus $\Delta t$ ) become smaller. To illustrate the convergence of the solutions using the described numerical scheme, the temperature course was calculated in two cases for which the exact solution was known:

(a) A sphere radius a in which the initial temperature is $\mathbf{T}_{\mathbf{0}}$ for $0<\mathrm{r}<\mathrm{b}$ and zero for $\mathrm{b}<\mathrm{r}<\mathrm{a}$. The temperature at $r=a$ remains zero at all times. No freezing oecurs. Under these conditions the temperature $T(r, t)$ is given by $(3)$,

$$
\begin{aligned}
T(r, t)=\frac{2 T_{0}}{r} \sum_{n=1}^{\infty} & \left\{\frac{a}{n^{2} \pi^{2}} \sin \left(\frac{n \pi b}{a}\right)\right. \\
\left.-\frac{b}{n \pi} \cos \left(\frac{n \pi b}{a}\right)\right\} & \sin \left(\frac{n \pi r}{a}\right) \\
& \times \exp \left\{\frac{-k^{2} n^{2} \pi^{2} t}{a^{2}}\right\} .
\end{aligned}
$$

The analytical solution has been calculated for $b=\frac{1}{2} a$ by a numerical approximation of $\mathrm{Eq}$. (19). Numerical solutions have been obtained by using the Eq. (9), (10), (13), and (14) for different values of $N_{s} / N_{\mathrm{I}}$, respectively, namely $10 / 5,20 / 10$,
$30 / 15,40 / 20$, and $60 / 30$. For one arbitrary chosen position $r=0.2 b$ the time needed to reach the temperature $0.35 T_{0}$ at this location was calculated for these different values of $N_{2}$ and $N_{1}$. Figure 10a shows the results of these computations.

The accuracy of the computed time using the analytical solution is about $0.5 \%$. Figure $10 a$ shows that with increasing number of shells the time in which the tempera.ture has reached $0.35 T_{o}$ decreases. This effect must be due to the use of Eq. (13). If $\Delta r$ is replaced by $\Delta r / K_{\mathrm{e}}$ c.q. $\Delta r / K_{a}$ it was observed from results of numerical analysis that the temperature profile at $r=b$ is concave. However, using Eq. (13) the temperature at $r=b$ is calculated by a linear interpolation which produces a too high temperature at $r=b$. This implies an a priori too slow numerical solution. Obviously, the error introduced in this way decreases rapidly with increasing number of shells.

The analytical solution together with the numerical solution with $N_{2}=60$ and $N_{1}=30$ are shown in Fig. 10 b. It was observed from numerical analysis that the same behavior was followed in the case that $a / b=10 / 8$. In that case only the errors were much smaller.

(b) Solidification in one dimension. Consider a liquid, bounded on one side by an infinite plane surface and not bounded in other directions. At time $t=0$ the liquid has a constant initial temperature $T_{1}\left(T_{1}>T_{f}\right)$, while the surface temperature remains constant $T_{2}$ at all times $\left(T_{y}<T_{f}\right)$. Frank and Von Mises (7) calculated the temperature as a function of time on different locations for $T_{1}=+4^{\circ} \mathrm{C}$, $T_{2}=-4^{\circ} \mathrm{C}$, and $T_{f}=0^{\circ} \mathrm{C}$. The liquid has heat transfer properties of water. The solid those of ice. In our numerical scheme the Eqs. (9), (10), and (12) are adjusted to the one dimensional problem. However, one has to keep in mind that the liquid is bounded on two sides and the thickness of the slab is $2 a$. The difference between these two cases is negligible as long as the 


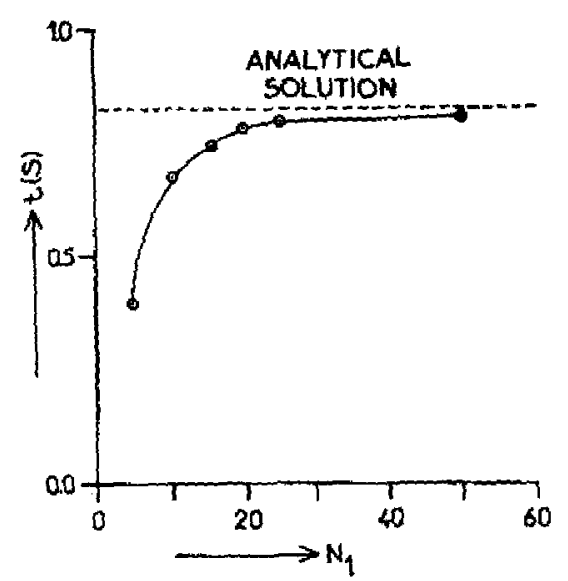

Fra. 1la. The time needed for the maving boundary to penetrate at a depth of $0.2 \mathrm{~mm}$ as a function of the number of shells. The dotted line indicates this time caleulated using the analytical solution.

temperature in the center of the slab remains near $T_{1}$. Again, calculations were carried out for different values of $N_{2}=N_{1}$, namely $5,10,15,20,25$, and 50 . The value of a was taken $1 \mathrm{~mm}$. The time needed for the moving boundary to penetrate at a depth of $0.2 \mathrm{~mm}$ was calculated for these different values of $N_{1}$. Figure 11 a shows the results of these computations. The accuracy of the computed time using the solution given by Frank and Von Mises ( 7 ) is about $0.5 \%$. Figure 11 a shows that with an increasing number of shells, the velocity of the moving boundary decreases.

This effect must be due to Eq. (15) in which the latent heat liberated by freezing of the thin shell between the outer surface and position $x_{\text {tayt }}$ is neglected. However, this error decreases rapidly with increasing number of shells. The analytical solution together with the numerical solution for $N_{2}=N_{1}=50$ are shown in Fig. $11 b$.

\section{Nongrowth of Computational Errors}

The problem of the solidification in one dimension can also be used to illustrate that the eomputational exrors are depressed in our numerical sehetne. During the iteration process an artificial error was introduced on time $t=0.42 \mathrm{~s}$ by changing the value of

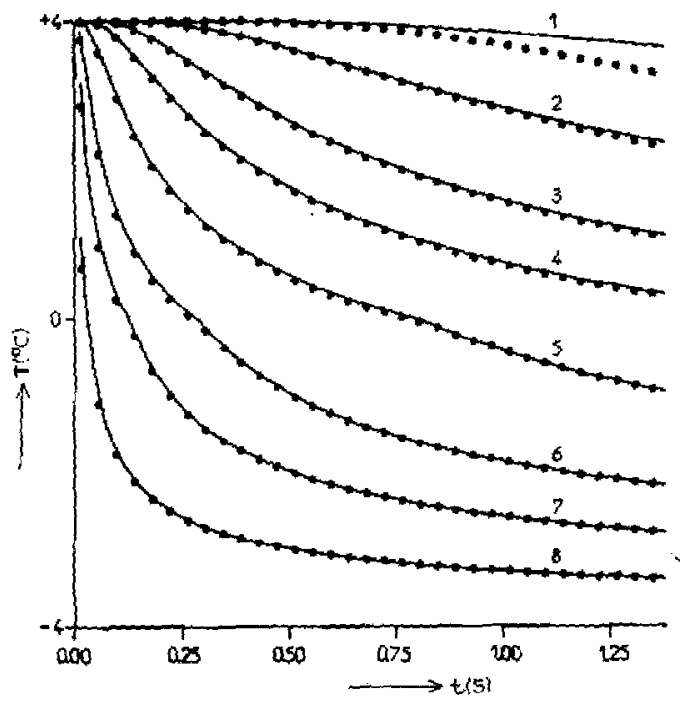

Frs. 11b. The aualytioal solution (line) together with the numerical solution (dots) with the parameters $N_{2}=N_{1}=50$ at different locations inside the slab with thickness $2 \mathrm{~nm}$; (1) $r=0.0 \mathrm{~mm}$ (2) $r=0.4 \mathrm{~mm}$; (3) $r=0.6 \mathrm{~mm}$; (4) $r=0.7 \mathrm{~mm}$; (5) $r=0.8 \mathrm{~mm} ;$ (6) $r=0.88 \mathrm{~mm} ;(7)^{*}=0.92$ mon; (8) $r=0.96 \mathrm{~mm}$.

$p$ from 1.11 to 1.99 in shell number 43 $\left(N_{1}=N_{2}=50\right)$. The effect of this arti-

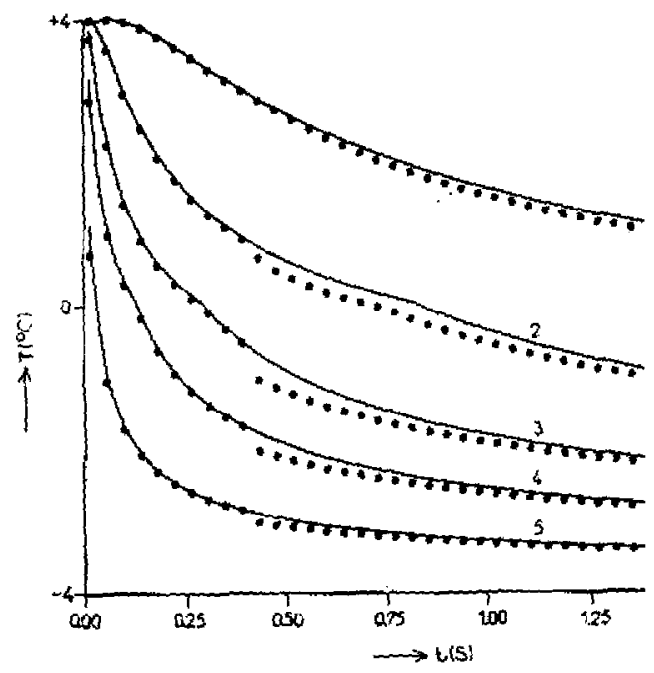

Fro. 12. Fffect of the artificially introduced error at time $t=0.43 \mathrm{~s}$ by changing the value of $p$ from 1.11 to $1.99 \mathrm{in}$ shell No. $43 .\left(N_{1}-N_{2}=50\right)$. The time dependent courses at different Jacations ars shown: (1) $r=0.6 \mathrm{~mm}$; (2) $r=0.8 \mathrm{~mm}$; (3) $r=0.88 \mathrm{~mm} ;$ (4) $r=0.92 \mathrm{~mm} ;(5) r=0.96 \mathrm{~mm}$. 


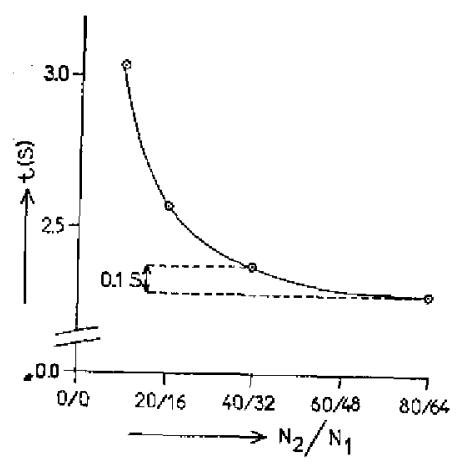

Fig. 13. The time needed for the moving boundary to reach the center of the sphere (diameter $2 \mathrm{~mm}$ ) as a function of the number of the shells.

ficially introduced error is illustrated in Fig. 12.

Obviously, the error does not grow during further iteration but even the numerical solution shows the tendency to move toward the analytical solution.

Analysis concerning convergence was also done for the numerical scheme used for the calculations on the freezing sphere. A specific situation $\left(N_{2} / N_{1}=1.2\right)$ was analyzed to some extent. For different values of $N_{2} / N_{1}$, namely $10 / 8,20 / 16,40 / 32$, and $80 / 64$, the time needed for the phase transition to reach the center of the sphere were calculated for these values. The results are shown in Fig. 13.

Comparing Figs. 11a, 11b, and 13 the same course is observed. If $\Delta t_{1}$ is the time difference produced by increasing the number of shells from $\left(N_{2}, N_{1}\right)$ until $\left(2 N_{2}, 2 N_{1}\right)$ and $\Delta t_{2}$ is the time difference produced by increasing the number of shells from $\left(2 N_{2}, 2 N_{1}\right)$ until $\left(4 N_{2}, 4 N_{1}\right)$ the figures show that:

$$
\Delta t_{2} / \Delta t_{1} \simeq \frac{1}{2}
$$

This implies that $\Delta t_{1}$ is an estimate of the difference between the numerical solution with parameters $2 N_{2}$ and $2 N_{1}$ and the solution which would be obtained in the case that $\Delta r \rightarrow 0$.

The fact that the eurve in Fig. 13 is the same as those of the Figs. 11a and 11b is not surprising because the numerical scheme used for the calculations on the freezing sphere is in fact a combination of the scheme used for the calculations on the cooling sphere without freezing and the scheme used for the solidification of the slab. Because of the depression of computational errors, the combination of the two schemes will not give rise to a growing crror.

Summarizing these findings it may be concluded that the computed solution for the freezing sphere with parameters $N_{2}=80$ and $N_{1}=64$ does not differ by more than $5 \%$ from the solution obtained for $\Delta r \rightarrow 0$. (Fig. 13). The same order of magnitude may be expected for the error in the solution for the parameters $N_{2}=62$ and $N_{1}=60$.

\section{ACKNOWLEDGMENTS}

Our thanks are due to Prof. J. J. Denier van der Gon for ugeful discussion and comments, Furthermore, we wish to thank José Bijvelt for skillul technical assistance.

\section{REFERENCFS}

1. Bachmann, L, and Smitt, W. W. Improved cryofixation applicable to freeze etching. Proc. Nall. Acad. Sci. U.S.A. 68, 2149 (1971).

2. Bank, H., and Mazur, P. Vistalization of freezing damage. J. Cell. Biol 57, 729 (1973).

3. Carslaw, H. S., and Jaeger, J. C. "Conduction of Heat in Solids," 2nd ed., Oxford Clarendon Press, 1959.

4. Chuang, J. K., and Szekely, J. The use of Green's functions for solving melting or solidification problems in the cylindrical coordinate system. Int. J. Heat Mass Transfer 15, 1171 (1972).

5. Crank, J. Two methods for the numerical solttion of moving boundary problems in diffusion and heat flow. Quart. $J$. Mech. and Appl. Math. 10, 220 (1957).

6. Elias, H, Hennig, A., and Schwartz, D. E. Stereology: Applications to biomedical research. Physiolog. Rey. 51, 158 (1971).

7. Frank, P., and Von Mises, R. "Die Differential und Intergralgleichungen der Mechanik und Physik, Heft 2," 3rd ed., Dover, New York, 1961 .

8. Heitz, W. L., and Westwater, J. W. Extension of the numerieal method for melting and freezing problems. Int. J. Heat Mass Transfer 13, 1371 (1970). 
9. Kreith, F., and Romie, F. E. A study of the thermal diffusion equation with boundary conditions corresponding to solidification or melting of materials initially at the fusion temperature. Proc. Roy. Soc. 68B 277 (1955).

10. Langford, D. A closed-form solution for the constant velocity solidification of spheres initially at the fusion temperature. Brit. $J$. Appl. Phys. 17, 286 (1966)

11. Lederman, J. M, and Boley, B. A. Axisymmetric melting or solidification of circular cylinders. Int. J. Heat Mass Transfer 13, 413 (1970).

12. Mazur, P. Kineties of water loss from cells at subzero temperatures and the likelihood of intracellular freezing. $J$. Gen. Physiol. 47,347 (1963).

13. Mazur, P. Cryobiology : The freezing of biological systens. Science 168. 939 (1970).

14. Meryman, H. T. Absence of unfrozen freezable water in rapidly frozen red cells. Cryobiology 7, 252 (1971).

15. Meryman, H. T. Osmotic stress as a mechanism of freezing injury. Cryobiology 8, 489 (1971).

16. Moor, H. Die Gerfrier-fixation lebender Zellen und ihre Anwendung in der Elektronenmikroskopie. Z. filr Zellforsch, 62, 546 (1964).

17. Moor, H. Recent Progress in the freeze-etching technique Phil. Trans, Roy. Soc. B261, 121 (1971).

18. Riehle, W. Ueber die Vitrifizierung verdünnter wässriger Lösungen $\mathrm{Ph}, \mathrm{D}$. Thesis, Zürich, 1968.

19. Staehelin, L. A, and Bertaud, W. S. Temperature and contamination dependent freeze etch images of frozen water and glycerol solutions. J. Ultrastruct. Res. 37, 146 (1971).

20. Sung, Hwan Cho, and Sunderland, J. Phase change in spherical bodies. Int. $J$. Heat Mass Transfer 13, 1231 (1970).

21. Underwood, E. E. Stereology, or the quantitative evaluation of microstructures. J. Microscop. 89, 161 (1969).

22. Williamson, E. D., and Adams, L. H. Temperature Distribution in solids during heating or cooling. Phys. Rev. 14, 99 (1919).

23. Zingsheim, H. P. Membrane structure and electronmicroscopy. The significance of physical problems and techniques (freeze-etching). Biochim. Biophys. Acta 265, 339 (1972). 